\title{
CONVERSE OF A WELL KNOWN THEOREM ON INTEGRAL MEANS ${ }^{1}$
}

\author{
LEE DETMER THOMPSON
}

0 . Introduction. Let $f(x)$ be a real-valued function defined and integrable, in the sense of Lebesgue, in an open interval $I$ of real numbers. If, for $h \neq 0$, the points $x, x+h$ lie in $I$, the integral of $f$ from $x$ to $x+h$, divided by $h$, is called the integral mean of length $h$ of $f$ at $x$, and will be denoted by $M_{h} f(x)$.

The integral mean of a function has been a useful tool in various investigations due to the fact that it is "smoother" than the function itself; for example, if $f$ is integrable in $I$, its mean will be continuous, ${ }^{2}$ while if $f$ has a continuous derivative of order $n \geqq 0$, the mean will have a continuous derivative of order at least $n+1$.

The theorem which will be proved is the converse, in a sense, of the above result. We shall show, under a certain restriction (that some restriction is required is evident from simple examples), that the smoothing power of the operation of taking the integral mean is limited; i.e., if $f$ is integrable but not continuous, $M_{h} f$ will be continuous but not continuously differentiable, while if $f$ has a continuous derivative of order at most $n, M_{h} f$ will have a continuous derivative of order at most $n+1$.

1. Differences, difference quotients, and means. Frequent use will be made of the difference, difference quotient, and mean operators, together with certain commutativity relations satisfied by them.

Let the difference of length $h$ of a function $f$ at $x$ be given by

$$
\Delta_{h} f(x)=\Delta f(x, x+h)=f(x+h)-f(x)
$$

and the difference quotient operator of length $h$ at $x$ by

$$
D_{h} f(x)=D f(x, x+h)=\frac{f(x+h)-f(x)}{h} .
$$

The mean of length $h$ of an integrable function $f$ at $x$ is defined by the formula

Received by the editors October 6, 1952.

1 This paper comes from a thesis submitted to the Graduate School of Indiana University in partial fulfilment of the requirements for the degree of Doctor of Philosophy. The author wishes to express her gratitude to Professor W. Gustin for his kindness and guidance in its preparation.

${ }^{2}$ It is to be understood in all that follows that a condition on $M_{h} f$ applies in the subinterval of $I$ in which it is defined. 


$$
M_{h} f(x)=\frac{1}{h} \int_{x}^{x+h} f(\theta) d \theta .
$$

Clearly, each operator of length $h$ commutes with all operators of length $k$ in the subinterval of definition of the function $f$ in which the iterated operators are defined. In addition, it is easily verified that

$$
D_{h} M_{k}=D_{k} M_{h} \text {. }
$$

If the function $f$ is continuous, the relations

$$
\begin{aligned}
& \lim _{h} \Delta_{h} f=0, \\
& \lim _{h} M_{h} f=f
\end{aligned}
$$

hold in $I$. By relations (4) and (6)

$$
D M_{h} f=\lim _{\boldsymbol{k}} D_{k} M_{h} f=\lim _{\boldsymbol{k}} D_{h} M_{k} f=D_{h} \lim _{\boldsymbol{k}} M_{k} f=D_{h} f .
$$

Therefore, if $f$ is continuous,

$$
D M_{h} f=D_{h} f .
$$

Suppose $D f$ exists and is continuous in $I$. Then from the fundamental theorem of integral calculus, it follows, for $x, x+h$ in $I$, that

$$
\int_{x}^{x+h} D f(\theta) d \theta=\Delta_{h} f(x)=h D_{h} f(x) .
$$

Therefore, using relations (3) and (7),

$$
M_{h} D f=D M_{h} f
$$

if $D f$ is continuous.

2. On the first derivative of the mean. Relation (7) of $\$ 1$ shows that if $f$ is continuous in $I$, the derivative of the mean exists and is continuous. Relation (7) still holds, however, if the assumption of continuity of $f$ is replaced by the weaker hypothesis that $\lim _{h} M_{h} f=f$. A function $f$ satisfying in the interval $I$ this weaker hypothesis will be called mean continuous (MC) in $I$.

It is for MC functions that the theorem stated in the introduction will be proved. The assumption of mean continuity is a natural one to make in view of the fact that relation (7), which is basic to the proofs that follow, holds in its presence. In addition, the theorem is false if the function $f$ is assumed only to be integrable. To see this, one need merely consider any discontinuous function which is con- 
stant almost everywhere.

If $f$ is $\mathrm{MC}$ in $I$ and for all $h \neq 0$ the derivative $D M_{h} f$ is continuous, then by relation (7), $D_{h} f$, and thus $\Delta_{h} f$, is continuous. Consider the identity

$$
\Delta_{k} \Delta_{h} f(x)+\Delta_{k} f(x)=\Delta_{k} f(x+h) .
$$

Since, by (5), $\lim _{k} \Delta_{k} \Delta_{h} f(x)=0$, it is clear that if $\lim _{k} \Delta_{k} f(x)=0$ for a single point $x$ in $I$, then $\lim _{k} \Delta_{k} f(y)=0$ for every point $y=x+h$ in $I$. Thus $f$ is either continuous in $I$ or discontinuous at every point in $I$. Since $\lim _{h} M_{h} f=f$, Baire's theorem on the limit of continuous functions guarantees that $f$ is continuous on a set of second category. Therefore, an MC function $f$ whose mean has, for all $h \neq 0$, a continuous derivative $D M_{h} f$ is continuous.

3. On the second derivative of the mean. Suppose $f$ is MC in $I$ and, for all $h \neq 0$, the second derivative of the mean $D^{2} M_{h} f$ exists and is finite. Consider the identity

$$
D_{k} \Delta_{h} f(x)+D_{k} f(x)=D_{k} f(x+h) .
$$

Since, by relation $(7)$,

$$
\lim _{\boldsymbol{k}} D_{k} \Delta_{h} f(x)=h \lim _{\boldsymbol{k}} D_{k} D_{h} f=h \lim _{\boldsymbol{k}} D_{k} D M_{h} f=h D^{2} M_{h} f
$$

exists for all $h$, whenever $x, x+h$ are in $I$, it follows from (10) that if $f$ has one of its unilateral derivates, say the upper right derivate ${ }^{*} D^{+} f=\lim \sup _{h \rightarrow 0+} D_{h} f$, finite at a point $x$, then ${ }^{*} D^{+} f(y)$ is finite for every $y=x+h$ in $I$. If one of the derivates of $f$ is infinite at a single point in $I$, it must be infinite of the same sign everywhere in $I$. Moreover, if $D f$ exists at a single point in $I, f$ is derivable in $I$ and either $D f$ is finite or infinite of the same sign everywhere in $I$.

A finite function $f$ possessing this behavior will be called a same derivative behavior, or SDB, function in $I$. To summarize, a finite function $f$ is an SDB function in $I$ if

(i) each unilateral derivate of $f$ is either finite in $I$ or infinite of the same sign in $I$;

(ii) whenever $D f$ exists at a single point, $f$ is differentiable in $I$, and $D f$ is either finite throughout $I$ or infinite of the same sign throughout $I$.

An SDB function for which all the unilateral derivates are infinite will be called an extreme function. For extreme functions $f$,

$$
{ }^{*} D^{+} f={ }^{*} D-f=+\infty, \quad{ }_{*} D^{+} f={ }_{*} D-f=-\infty .
$$

Indeed, if one of the upper derivates, say ${ }^{*} D^{+} f$, satisfies ${ }^{*} D^{+} f=-\infty$, 
then ${ }^{*} D^{+} f \leqq * D^{+} f$ implies that $f$ has an infinite right derivative everywhere in $I$. It is known, however, that a finite function $f$ can have an infinite unilateral derivative on at most a set of measure zero [3]. ${ }^{3}$ Thus it is also clear that if an SDB function has a derivative in $I$, that derivative is finite in $I$.

We shall prove in $\$ 5$ that an SDB function is either differentiable or extreme. Further, we shall show that a continuous SDB function cannot be extreme, hence is differentiable.

Making use of these results on SDB functions, it is easy to prove that if $f$ is $\mathrm{MC}$ in $I$ and, for all $h \neq 0$, the second derivative of the mean $D^{2} M_{h} f$ exists and is continuous, then $D f$ exists and is continuous in $I$. Since $D^{2} M_{h} f$ exists, relation (10) shows that $f$ is an SDB function. Moreover, since $D M_{h} f$ must be continuous, the result of $\$ 2$ shows that $f$ is continuous. Thus $f$, being a continuous SDB function, is differentiable. To see that $D f$ is continuous, note that $D f$ is the limit of the continuous functions $D_{h} f$, while

$$
h D^{2} M_{h} f=h D D_{h} f=h D_{h} D f=\Delta_{h} D f
$$

is continuous, and therefore the situation is analogous to that occurring in $\$ 2$.

4. On the $n$th derivative of the mean. Using the results of $\$ \$ 2$ and 3 , the main theorem is proved by induction.

MAIN Theorem. Let the integrable function $f$ be $M C$ in the open interval $I$. Then if, for all $h \neq 0, M_{h} f$ has a continuous derivative of order $n+1, n \geqq 0, f$ has a continuous derivative of order $n$ in $I$.

Proof. The truth of the theorem for $n=0,1$ has been established in $\S \S 2,3$. Assume the theorem is true for $n-1 \geqq 0$ : to prove it for $n$. If for all $h \neq 0, D^{n+1} M_{h} f$ is continuous, it follows that $D^{2} M_{h} f$ is continuous, and therefore $D f$ is continuous in $I$. By virtue of relation (8),

$$
D^{n+1} M_{h} f=D^{n}\left(D M_{h} f\right)=D^{n} M_{h} D f .
$$

Thus $D f$ is MC while, for all $h \neq 0$, its mean $M_{h} D f$ has a continuous derivative of order $n$. By assumption, $D^{n-1}(D f)=D^{n} f$ exists and is continuous in $I$, so by induction the theorem is proved.

\section{Theorems on SDB functions.}

THeOREm 1. If $f$ is an $S D B$ function in $I, f$ is extreme or $f$ is differentiable in $I$.

In order to prove Theorem 1 we make use of the following

\footnotetext{
${ }^{3}$ Numbers in brackets refer to the bibliography.
} 
LEMмA. If a finite function $f$ defined on an open interval $I$ has its upper right derivate ${ }^{*} D+f<\infty$ on $I$, then its upper derivate ${ }^{*} D f$ $=\lim _{\sup _{h \rightarrow 0}} D_{h} f$ is uniformly bounded from above on some open subinterval of $I$.

Proof. For each point $x$ in $I$, let $I_{n}(x)$ be that open subinterval of $I$ consisting of all points $y>x$ in $I$ which are closer than $1 / n$ to $x$. Let $F_{n}$ be the set of points $x$ of $I$ such that

$$
D f(x, y)=\frac{f(y)-f(x)}{y-x} \leqq n
$$

for every $y$ in $I_{n}(x)$. We shall show that $F_{n}$ is closed from the right.

Suppose, to the contrary, that there exist points $x_{m}, x$ in $I$ with $x_{m} \rightarrow x^{+}, x_{m}$ in $F_{n}$, but $x$ not in $F_{n}$. Therefore, $D f(x, y)>n$ for some point $y$ in $I_{n}(x)$, and so has the value $n+\epsilon, \epsilon>0$. Moreover since $y$ lies in $I_{n}(x)$ and $x_{m} \rightarrow x^{+}, y$ also lies in $I_{n}\left(x_{m}\right)$ for almost all $m$. Hence for all such $m$, we have $D f\left(x_{m}, y\right) \leqq n$ since $x_{m}$ belongs to $F_{n}$. Now

$$
f\left(x_{m}\right)-f(x)=[f(y)-f(x)]-\left[f(y)-f\left(x_{m}\right)\right]
$$

or

$$
\begin{aligned}
\Delta f\left(x, x_{m}\right) & =\Delta f(x, y)-\Delta f\left(x_{m}, y\right) \\
& =(y-x) D f(x, y)-\left(y-x_{m}\right) D f\left(x_{m}, y\right) .
\end{aligned}
$$

Therefore, for almost all $m$,

$$
\Delta f\left(x, x_{m}\right) \geqq(y-x)(n+\epsilon)-(y-x) n=(y-x) \epsilon,
$$

whereupon

$$
{ }^{*} D^{+} f(x) \geqq \lim _{m} D f\left(x, x_{m}\right)=+\infty,
$$

contrary to ${ }^{*} D^{+} f(x)<+\infty$. This proves $F_{n}$ closed from the right.

Every point $x$ in $I$ is in some set $F_{n}$. If this were not the case, some $x$ in $I$ would belong to no set $F_{n}$; thus for each $n$, there would be a point $y_{n}$ in $I_{n}(x)$ with $D f\left(x, y_{n}\right)>n$, whereupon, since $y_{n} \rightarrow x^{+}$,

$$
{ }^{*} D^{+} f(x) \geqq \lim _{n} D f\left(x, y_{n}\right)=+\infty
$$

contradicting ${ }^{*} D^{+} f(x)<+\infty$. Thus $I$ is the union of the sets $F_{n}$. According to Baire's theorem, at least one of the sets $F_{n}$ must be somewhere dense-dense, say, in the open subinterval $J$ of $I$. But this set $F_{n}$, being closed from the right, must contain $J$. Consequently, 
for any two distinct points $x, y$ of $J$ closer than $1 / n$ we have $D f(x, y)$ $\leqq n$, so

$$
{ }^{*} D f(x)=\limsup _{y \rightarrow x} D f(x, y) \leqq n
$$

for every $x$ in $J$. This completes the proof of the lemma.

Proof of Theorem 1. If $f$ is not extreme, one of its derivates, say ${ }^{*} D^{+} f$, is finite in $I$, Under this condition, it has been proved (A. Denjoy [1] for continuous functions; S. Saks [2] for arbitrary finite functions) that ${ }^{*} D^{+} f={ }_{*} D-f$ almost everywhere in $I$. The preceding lemma shows that ${ }^{*} D-f$ is also finite in $I$, whence ${ }^{*} D-f={ }_{*} D^{+} f$ almost everywhere. Thus $D f$ exists and is finite almost everywhere in $I$, and $f$, assumed to be a nonextreme SDB function, must therefore be differentiable.

By virtue of Theorem 1, it is necessary only to prove that a continuous function cannot be extreme in order to establish

TheOREM 2. If $f$ is a continuous $S D B$ function in $I, f$ is differentiable in $I$.

Proof. Let $a, b$ be any two points in $I$. The continuous function

$$
g(x)=f(x)-(x-a) D f(a, b)
$$

has the same differentiability properties as $f$. Since $g(a)=g(b), g$ either is constant in the interval $[a, b]$ or assumes an extreme value at some point $y$ in the interior. Thus one, at least, of the upper derivates of $g$ at $y$ is nonpositive, and $g$ cannot be extreme.

\section{BIBLIOGRAPHY}

1. A. Denjoy, Sur les nombres dérives des fonctions continues, J. Math. Pures Appl. vol. 80 (1915) pp. 105-240.

2. S. Saks, Sur les nombres dérives des fonctions, Fund. Math. vol. 5 (1924) pp. 98104.

3. - Theory of the integral, New York, Hafner, 1937, Chapter IX.

INDIANA UNIVERSITY 